Article

\title{
Strain Rate of Metal Deformation in the Machining Process from a Fluid Flow Perspective
}

\author{
Keguo Zhang ${ }^{1,2, *}$, Keyi Wang ${ }^{1}$, Zhanqiang Liu ${ }^{2, *}$ and Xiaodong $\mathrm{Xu}^{1}$ \\ 1 School of Mechanical, Electrical and Information Engineering, Shandong University, Weihai 264209, China \\ 2 Key Laboratory of High Efficiency and Clean Mechanical Manufacture, Shandong University, \\ Ministry of Education, Jinan 250061, China \\ * Correspondence: zkg@sdu.edu.cn (K.Z.); melius@sdu.edu.cn (Z.L.)
}

Received: 11 March 2020; Accepted: 23 April 2020; Published: 27 April 2020

\begin{abstract}
Metal cutting speeds are getting faster with the development of high-speed cutting technology, and with the increase in cutting speed, the strain rate will become larger, which makes the study of the metal cutting process more inconvenient. At the same time, with the increase in strain rate, the dislocation movement controlling the plastic deformation mechanism of metal will change from thermal activation to a damping mechanism, which makes the metal deformation behave more like a fluid. Therefore, it is necessary to explore new ways of studying machining from the perspective of fluid flow. Based on this, a fluid model of the metal cutting process is established, and a method for calculating the strain rate is proposed from the point of view of flow. The results of the simulation and measurements are compared and analyzed. The results show that the strain rate on the rake face will be affected by the friction between the chip and tool; the nearer the distance between the chip layer and tool rake face, the bigger the strain rate will be. The strain rate in the central shear plane is much larger than in other areas along the shear plane direction, and in which two ends are the biggest. It can achieve rougher, quantitative research. This shows it is feasible to study machining from the viewpoint of fluid flow, though it still needs a lot of theoretical support and experimental confirmation.
\end{abstract}

Keywords: machining; dislocation; strain rates; fluid flow; deformation mechanism

\section{Introduction}

Correctly describing the mechanical behavior of metal materials during the deformation process under different strain rate conditions is an important subject of solid mechanics. Campbell [1] divided the strain rates into three categories: low strain rate, $10^{-4}-10^{-1} \mathrm{~s}^{-1}$; middle strain rate, $10^{-1}-10^{2} \mathrm{~s}^{-1}$; and high strain rate, $10^{2}-10^{4} \mathrm{~s}^{-1}$. Metal deformation will show different behaviors under different strain rates, such as elasticity, plasticity or viscosity [2]. The material response at a low or medium strain rate is usually described by one behavior, elasticity or plasticity, or a combination of the two. With increasing strain rate, the metal deformation form will change from static to dynamic behavior. The effects of material viscosity should be considered because it describes the viscous behavior of the materials in terms of the relevant strain rate (strain rate includes time factors). Regardless of ductile fracture or brittle fracture, it is a process that develops at a limited rate. Therefore, the mechanical properties of the materials are essentially strain-rate dependent. However, the relevant rate cannot accurately describe the viscous behavior of materials at high strain rates.

Machining involves large plastic deformation; in this process, the strain rate increases up to $10^{5} \mathrm{~s}^{-1}$ orders of magnitude with increasing cutting speed [3,4]. Studies of the cutting process usually focus on elastic-plastic deformation; as a result, the theoretical calculation results evidently differ from the 
experimental data. Therefore, the viscous behavior of material deformation during cutting is worthy of further study and exploration.

The influence of material viscosity on the deformation of materials under high strain rates has been increasingly investigated. Rusinek [5] studied the deformation behavior of aluminum under different strain rates and reported that viscous stress should be considered in evaluating the flow stress of materials. Kapoor [6] pointed out that the flow stress of a material is composed of three parts: thermal activation, athermal resistance and viscous damping parts. Dao-chun [7] studied the dynamic behavior and deformation mechanism of $\mathrm{C} 5191$ bronze under high strain rates. The results indicate that the dominant mechanism of plastic deformation is due to the increase and change in dislocation motion at different strain rates. The microscopic mechanism of plastic deformation is dislocation movement. In studies of dislocation motion, the effect of material viscosity is commonly considered. In computer simulations, the rate-dependent constitutive model is typically used to describe the viscosity of materials.

Bingham is one of the first scholars to study the viscosity of solid materials and establish a simple mechanical model. He developed a uniaxial material constitutive model under stress by using a combination of plastic and viscous components to describe the viscoplasticity properties. For viscoplastic materials, the yield conditions are not only related to stress, plastic strain and strengthening properties, but also to the parameters that reflect the viscosity of the material. The viscous characteristics of the materials are important in the case of explosive impact loading, where the strain rate of the material increases dramatically in a very short time and the dynamic mechanical response of the material is similar to that of the fluid. Therefore, many scholars have studied plastic deformation at high strain rates from the point of view of fluid flow.

Solids and fluids are two natural forms of matter in nature and are differentiated in terms of response to shear stress; in contrast to fluid, a solid can withstand certain shear forces. Under certain extreme conditions, solids may behave like fluids. The Deborah number, from the definition of rheology, points out the difference between a solid and a fluid and is represented as $D_{b}=$ time of relaxation/time of observation. If the time of relaxation of a material under observation is very small, the material flows. In the metal cutting process, the strain rate can reach $10^{5} \mathrm{~s}^{-1}$ and above, and the metal behavior is more like a fluid than a solid; thus, high speed machining can be better modeled as fluid flow than as solid.

Eugene studied the cutting process from the aspect of fluid flow, and designed an experimental apparatus to model the cutting process as the flow of a fluid around the tool tip [8] (Figure 1). Fluid is pumped into place A and flows past tools with different rake angles at B. Powdered bakelite is introduced at $C$ to make the streamlines visible, and photographs are taken by the camera at $D$. El-zahry [9] used Bingham liquid to model the plastic flow between the chip and rake face and study the friction of the second shearing zone. Kwon [10] presented a potential flow analysis approach in ultra-precision machining to predict the contact length and cutting forces. Müller [11] believes that the cutting fluid melt layer between the chip and the cutting edge induces the chip to easily flow, thereby decreasing the high-speed cutting force. The strain rate is very high during metal cutting, and the relationship between the shear stress and shear strain rate appears to be linear under high strain rate conditions because of the fluid characteristics [12,13]. According to this linear relationship, Kazban [14,15] modeled high-speed machining according to the potential flow problem of one source and two sinks; he considered the high-speed cutting process as a simple closed polygonal boundary flow and transformed the process into fluid flow issues. Some metals exhibit similar characteristics to fluids at room temperature. Sundaram [16] used a hard steel wedge plow to cut a copper surface and studied the sliding of the metal interface. The surface slip of copper showed laminar flow, and folding and laminar flow interruption occurred. Full-scale sinuous flow simulations were used by Vandana [17] to study metal cutting; he finds that folding and sinuous flow can occur even at a low friction and at very low cutting speeds. However, the reason why the relationship between the shear stress and shear strain rate is linear under high strain-rate conditions is unknown and must be studied with respect to 
the microscopic control mechanism of plastic deformation; this paper answers this question through dislocation motion analysis. Based on this theory, the strain-rate distribution in the cutting process is studied from the perspective of fluid flow by means of simulation and experimental verification, so as to provide new ideas for the research of the cutting process.

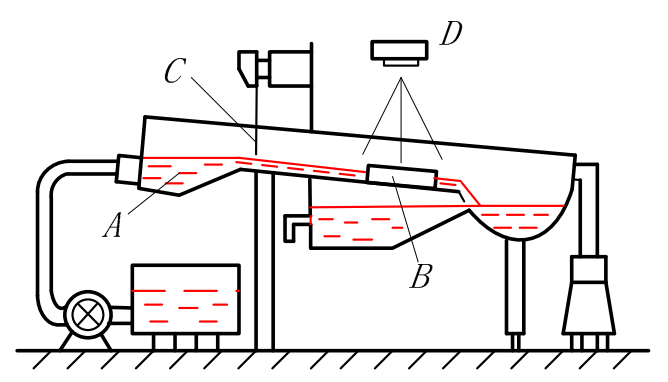

Figure 1. Apparatus used by Eugene (1952) [8]. A-Baffle chamber; B-Cutting tool; C-Baffle; D-Camera.

\section{Materials and Methods}

\subsection{Microscopic Foundation of Metal Cutting Research from a Fluid Flow Perspective}

The plastic deformation control mechanism has been studied for a long time. From a microscopic point of view, the plastic deformation of a metal material mainly involves the movement and proliferation of dislocations, including slip and twinning. However, the plastic deformation mechanism of metals is complex, as metal cutting is a process of dislocation accretion and annihilation. An increase in the density of movable dislocations will lead to work hardening. At the same time, dislocation movement leads to dislocation interaction, which makes the study of the plastic deformation mechanism more complicated. Slip is the main deformation form in the cutting process, so here we only study the plastic deformation mechanism from a dislocation slip aspect. The accepted mechanisms include heating activation, dislocation damping and the relativistic effect of the dislocations [18].

Dislocations can be multiplied through several mechanisms. According to the dislocation mechanism of the Frank-Read source (Figure 2), the dislocation line AB is pinned by dislocation nodes at both ends. The time needed to remove the node constraint is $t_{t}$, and the time spent by moving dislocation between the adjacent dislocation node is $t_{w}$. When the dislocation moves to the obstacle under applied stress, the dislocation will be successfully transformed to the other side of the obstacle by a certain frequency due to the influence of heat. The transition frequency is

$$
v=v_{0} \exp \left[-\frac{\left(E_{c}-\tau v^{*}\right)}{k T}\right]
$$

where $v_{0}$ is the vibration frequency of a dislocation heated near an obstacle, $E_{c}$ is the energy needed to overcome the short-range barrier, $\tau$ is the applied stress, $v^{*}$ is the thermal active volume, $k$ is the Boltzmann constant and $T$ is the temperature. Thus, the time spent by moving dislocation between the adjacent dislocation node is

$$
t_{t}=v^{-1}
$$

The moving dislocation will slip after removing the node pinning. The viscous damping $B$ of the dislocation should be considered when it slips in the barrier (slip speed $v_{s}=\tau b / B, \mathrm{~b}$ is the burgers vector; $B$ is the viscous damping). Assuming that the distance between the adjacent dislocation node is $L$, then the time required for the dislocation slip $L$ distance is

$$
t_{w}=\frac{L}{v_{s}}
$$


The speed of the dislocation that moves in distance $L$ is

$$
v_{d}=\frac{L}{t_{t}+t_{w}}
$$

According to the Orowan [19] equation $\dot{\gamma}=\rho b v_{d}$, the relationship between the stress and strain rate can be acquired:

$$
\dot{\gamma}=\rho b L /\left(v_{0}^{-1} \exp \left(\left(E_{c}-\tau v^{*}\right) / k T\right)+B L / \tau b\right)
$$

At the low strain-rate stages, usually $t_{t}>>t_{w}$, and the thermal activation mechanism is dominant in the plastic deformation process of the materials. The relationship between the stress and strain rate can be sampled as

$$
\dot{\gamma}=\rho b L k T v_{0} / \exp \left(\left(E_{c}-\tau v^{*}\right)\right.
$$

The strain rate increases with increasing cutting speed, and the shear yield stress increases due to the strain rate effect. $t_{t}, t_{w}$ will decrease; $t_{w}$ is inversely proportional to the yield stress; that is, $t_{t}$ follows an exponential decay with increasing yield stress and decays faster than $t_{w}$. A critical value exists to make the two parameters equal; consequently, $t_{t}<<t_{w}$. At this time, the damping mechanism of the dislocation motion is dominant, and the relationship between the stress and strain rate can be sampled as

$$
\tau=\frac{B}{\rho b^{2}} \dot{\gamma}=\eta \dot{\gamma}
$$

This equation represents the properties of fluid. The sensitivity of the material yield stress-to-strain rate will increase rapidly according to their linear relationship. Hence, research of the cutting process can start with fluids.

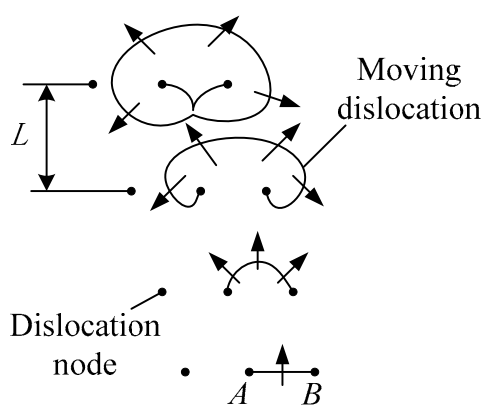

Figure 2. Multiplication mechanism of the dislocation source.

\subsection{Fluid Models for the Metal Cutting Process}

In studying the cutting process from the point of view of flow, the first step is to build a suitable cutting model that can correctly reflect the characteristics of the material being removed. The generation of chips is a flow process separated from the workpiece when the force of the removed material exceeds the yield limitation; as such, additional stress is not required for deformation. Kazban [14,15] pointed out that the potential flow solution can be used to understand the mechanics of machining at high speeds. Klocke [20] studied the temperature fields in metal cutting through the panel method of fluid mechanics. Zhang [21] analyzed the distributions of the strain rates by using computational fluid dynamics (CFD), which is based on Navier-Stokes equations. Assuming a material is incompressible and considering the mass conservation is low, then the cutting process can be modeled as

$$
u_{j} \frac{\partial u_{i}}{\partial x_{j}}=-\frac{1}{\rho} \frac{\partial p}{\partial x_{i}}+\frac{\mu}{\rho} \frac{\partial^{2} u_{i}}{\partial x_{j}^{2}}
$$


where $u$ is the material flow velocity, $\mu$ is the viscosity of the fluid, $\rho$ is the material density and $p$ is the local thermodynamic pressure.

The energy equation of this process can be expressed as

$$
\rho c \frac{D T}{D t}=\phi^{\prime}+\nabla \cdot(k \nabla T)
$$

where $c$ is the specific heat; $D / D t$ is the symbol of the total derivative; $\phi^{\prime}$ is the dissipation item, which mainly includes the plastic dissipation of the chips and heat dissipation caused by friction between the cutting tools and chips [22]; $k$ is the thermal conductivity; $\nabla$ is the symbol of grads; and $T$ is the temperature.

Solving the two equations can provide results that include the strain-rate distribution (Figure 3). The numbers marked in the figure are ordinal numbers arranged according to their true values, and the real value needs to be multiplied by $10^{3}$. The strain rate value in first deformation is the largest and gradually decreases from the center (shear plane) to the exterior. Additional details are presented in previous studies $[14,15,21]$. Although additional models can be established from the fluid-flow aspect, studying the metal cutting process is a new direction.

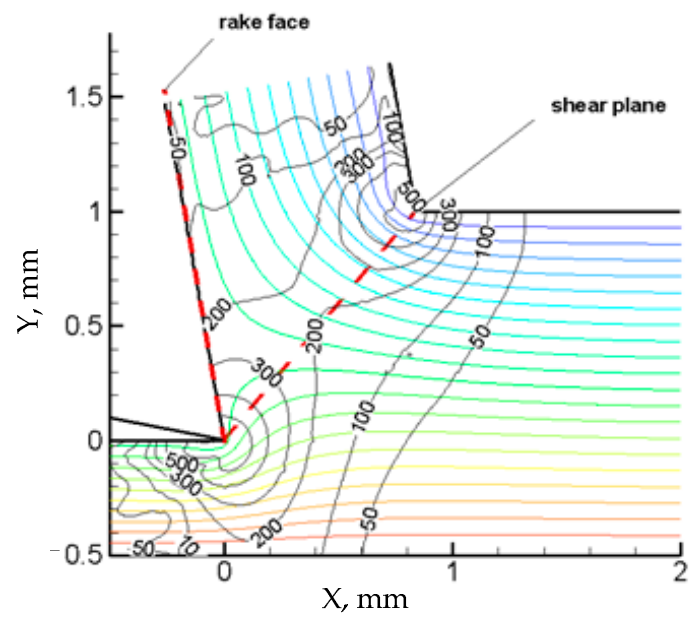

Figure 3. Strain rate distribution in the cutting process: 7050 aluminum alloy, $20 \mathrm{~m} / \mathrm{s}$ cutting speed.

\subsection{Strain Rate Calculations from a Flow Viewpoint}

Parameters considered in metal cutting include strain, strain rate and temperature. Scholars have investigated such parameters to understand their changing laws and applied them to engineering practice. The increase in cutting speed complicates the on-line measurement of physical parameters. An appropriate method must be developed for studying the cutting process parameters. Moreover, studying the strain rate and strain from the point of view of fluid flow is convenient and simple. The entire calculation process is shown in Figure 4.

First, tiny meshes are created on the surface of the workpiece, which has many small holes on the edge (Figure 4a). The chip root can be easily acquired because of the structure of the hole. As the milling feed increases, the material near the cutting tool side decreases until the chip root is separated from the workpiece due to the tool feed (Figure $4 \mathrm{~b}$ ). In the cutting process, the mesh will be distorted after flowing through the shear plane (Figure $4 \mathrm{c}$ ), where $V$ is the cutting velocity, $V_{\mathrm{c}}$ is the chip velocity along the direction of the rake face, $V_{S}$ is the chip slip velocity along the direction of the shear plane, $\alpha$ is the rake angle and $\phi$ is the shear angle. $X$ is set as the direction along the cutting tool rake face direction, and $Y$ is the direction along the vertical tool rake face direction. Assuming that the grid with a side length of $x_{n}$ moves $L_{x}$ distance after $t_{x}$ time, becoming $x_{n+1}$ with a deflected $\beta$ angle (Figure $4 \mathrm{~d}$ ), the strain along the $X$ direction can be obtained: 


$$
\varepsilon_{x}=\frac{\left|x_{n+1} \cos \beta-x_{n}\right|}{L_{x}}
$$

Strain rate along the cutting tool rake face direction can be expressed as

$$
\dot{\varepsilon}_{x}=\frac{\left|x_{n+1} \cos \beta-x_{n}\right|}{t_{x} L_{x}}
$$

According to the characteristics of fluid flow, after the first grid passes through the shear plane, the second grids will flow to the original location of the first grid. If adjacent meshes are measured (ignoring the width of the laser beam machining line), then the distance $L_{x}$ moving along direction $\mathrm{X}$ is approximately equal to the measurement length of the first mesh. Therefore, the time can be expressed as

$$
t_{x}=\frac{L_{x}}{V_{c}}=\frac{L_{x} \cos (\phi-\alpha)}{V \sin \phi}=\frac{x_{n} \cos (\phi-\alpha)}{V \sin \phi}
$$

Strain rate along the $\mathrm{X}$ direction can be expressed as

$$
\dot{\varepsilon}_{x}=\frac{\left|x_{n+1} \cos \beta-x_{n}\right| V \sin \phi}{x_{n}^{2} \cos (\phi-\alpha)}
$$

Strain rate along the $\mathrm{Y}$ direction can be obtained by the same principle

$$
\dot{\varepsilon}_{y}=\frac{\left|y_{n+1} \cos \beta-y_{n}\right| V \cos \alpha}{y_{n}^{2}}
$$

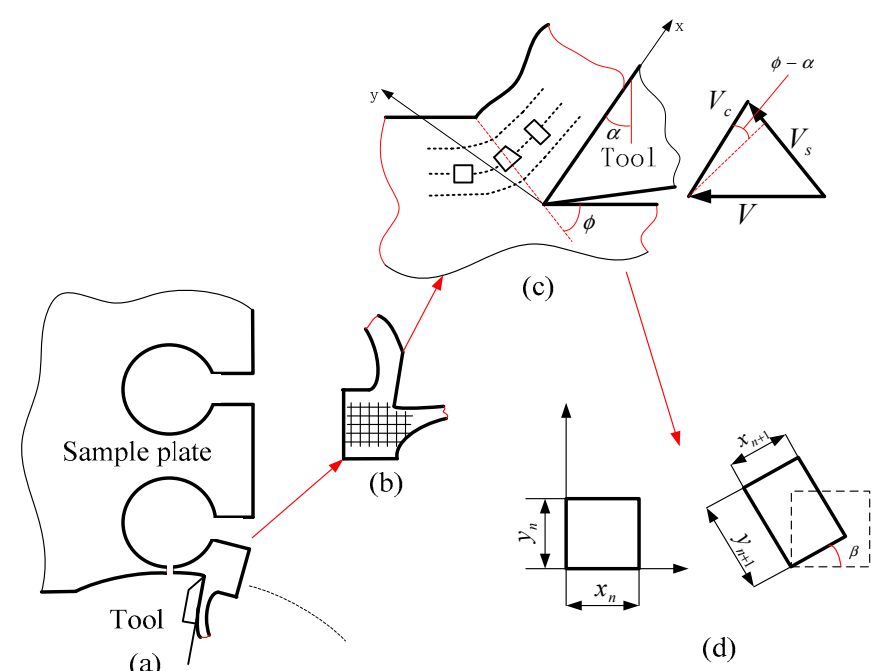

Figure 4. Method of strain rate calculation: (a) Mechanism of chip root acquisition; (b) chip root with deformed mesh; (c) flow of the chip and velocity vectors; (d) deformation of the mesh passing through the shear plane.

\subsection{Experimental Design}

The VMC850B vertical machining center (Beier Machine Tool Works Co. Ltd, Beijing, China) is equipped with a $160 \mathrm{~mm}$ in diameter groove-milling cutter head. The milling cutter adopts the KC725M-coated blade of Kennametal (Kennametal Inc., Latrobe, USA). Only one blade is installed to simulate the single-edge orthogonal cutting process; the removed workpiece is made of aluminum 7050 and machined into $2 \mathrm{~mm}$-thick plates. The $0.05 \mathrm{~mm} \times 0.05 \mathrm{~mm}$ mesh is pre-machined on the workpiece 
surface by femtosecond laser. Femtosecond laser processing can be used to avoid the influence of heat on the grid.

\section{Results and Discussion}

\subsection{Mesh Measurement Results}

The chip root with deformed meshes is indicated in Figures 5 and 6. Four streamlines are selected along the rake face direction, and the lengths of 10 meshes on each streamline are marked as $1-40$ (Figure 5). Three shear lines are taken along the shear plane direction, and 10 meshes are obtained from each shear line for measurement. These meshes are marked as 1-30 (Figure 6). The length of each primitive grid in the two directions is shown in Tables 1 and 2.

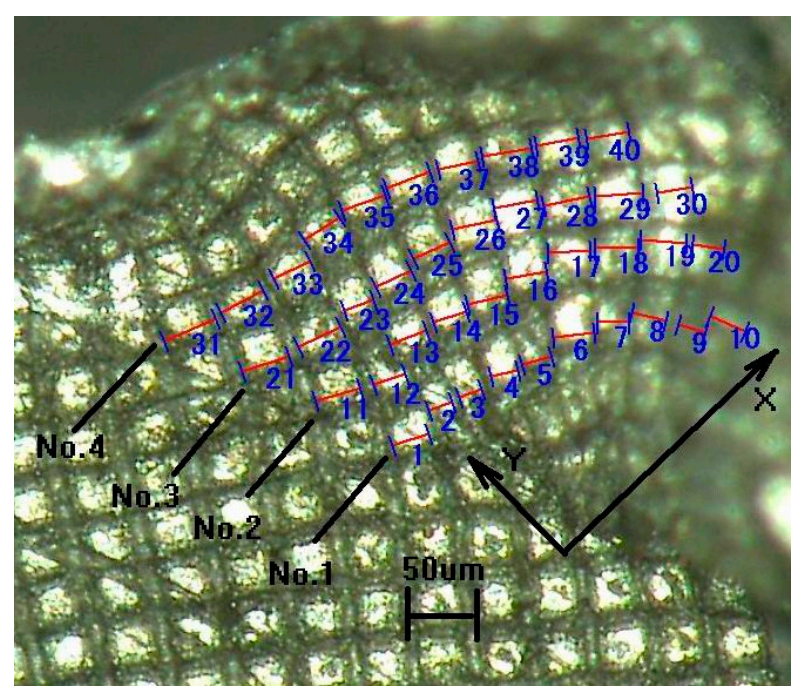

Figure 5. Mesh lengths along the direction of the rake face.

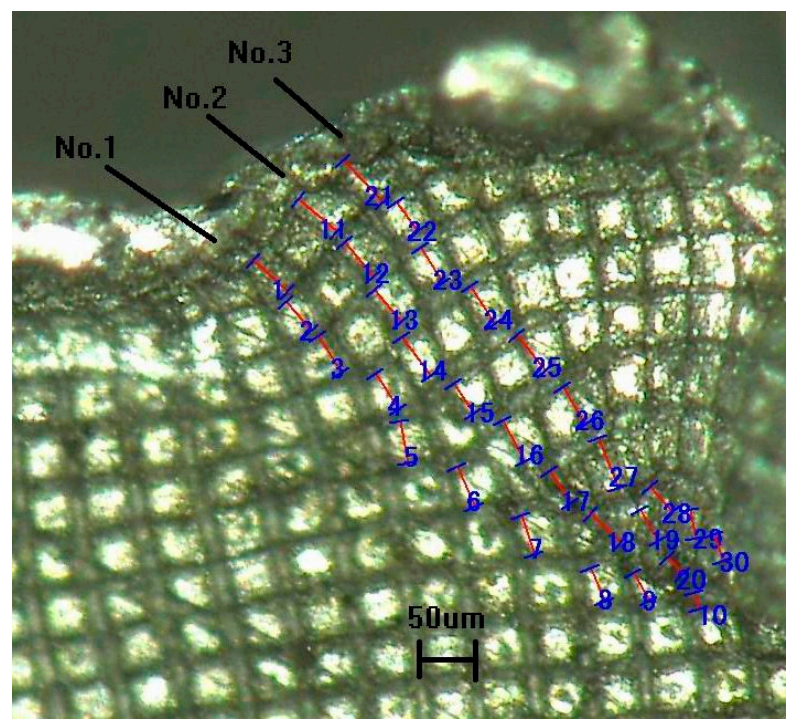

Figure 6. Mesh lengths along the direction of the shear plane. 
Table 1. Original lengths of the meshes along the rake face direction.

\begin{tabular}{ccccccccccc}
\hline Line & \multicolumn{10}{c}{ Length $(\mu m)$} \\
\hline No.1 & 31.05 & 20.2 & 19.3 & 22.43 & 24.74 & 33.58 & 25.73 & 28.43 & 22.91 & 31.16 \\
No.2 & 37.71 & 27.13 & 31.68 & 28.03 & 33.29 & 33.85 & 34.27 & 35.27 & 37.28 & 26.02 \\
No.3 & 40.09 & 37.95 & 25.33 & 31.55 & 32.42 & 37.01 & 35.59 & 37.95 & 39.18 & 28.99 \\
No.4 & 45.68 & 38.8 & 32.82 & 33.85 & 37.38 & 37.71 & 33.09 & 40.58 & 34.96 & 34.79 \\
\hline
\end{tabular}

Table 2. Original lengths of the meshes along the shear plane direction.

\begin{tabular}{ccccccccccc}
\hline Line & \multicolumn{10}{c}{ Length $(\mu m)$} \\
\hline No.1 & 39.82 & 33.09 & 37.28 & 36.48 & 36.83 & 37.55 & 35.27 & 31.16 & 27.31 & 14.8 \\
No.2 & 43.32 & 38.56 & 35.88 & 41.56 & 30.93 & 40.09 & 33.04 & 35.73 & 30.74 & 19.56 \\
No.3 & 49.89 & 30.69 & 35.97 & 38.6 & 39.3 & 37.6 & 46.19 & 29.05 & 25.24 & 24.11 \\
\hline
\end{tabular}

\subsection{Data Analysis and Discussion}

The strain rate distribution on each line can be calculated using Formulas (13) and (14). A single numerical value fluctuates greatly due to the influence of the front and rear grids; thus, studying the strain rate of a single grid is meaningless. The average values of each line are close to the actual distribution. The calculation results are shown in Figure 7 . The average strain rates of the streamline on the rake face are $1.53 \times 105,0.91 \times 105,0.89 \times 105$ and $0.52 \times 105 \mathrm{~s}^{-1}$. The average strain rates of the shear line on the shear plane direction are $0.54 \times 105,0.80 \times 105$ and $0.70 \times 105 \mathrm{~s}^{-1}$, respectively. As shown in Figure 7a, the farther away from the rake face, the smaller the value will be because of the friction between the rake face and chip; the greater the friction, the greater the deformation and the higher the average strain rate will be. Figure $7 \mathrm{~b}$ shows that the average strain rate on the second shear line is the largest because it is located at the center of the first deformation zone, and the other regions decrease in turn; this finding is similar to the calculation and simulation results [15,21].

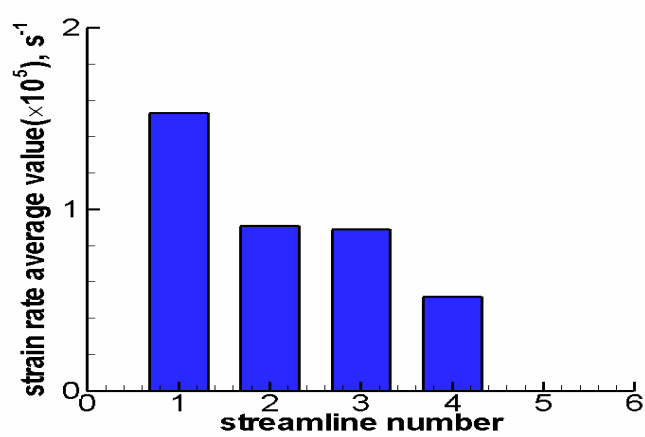

(a)

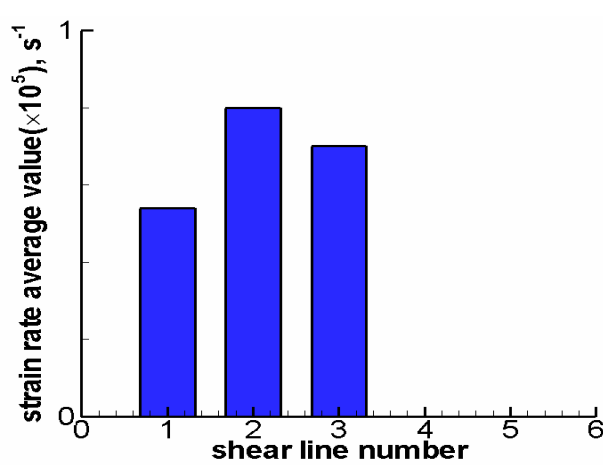

(b)

Figure 7. Average value of the strain rate (a) along the rake face direction and (b) along the shear plane direction.

The calculated results on the rake face and shear plane were compared with the experimental results (Figure 8). The experimental results include the first streamline (Figure 5, No.1) along the rake face direction and the second shear line (Figure 6, No.2) along the shear plane direction. The actual measured values may vary considerably because of the influence of various factors (such as cutting speed, material, etc.), but this phenomenon does not affect the study and analysis of the distribution law of the strain rate; for example, the results of the grid measurement with different cutting speeds will vary greatly, but the distribution rule of the strain rate will not change-that is, the strain rate in the center of the first deformation zone is larger than that in other regions along the shear plane direction, and the strain rate at both ends of the shear plane is the largest, with the values decreasing sequentially outward. The measurement results of the different samples may also change at the same 
cutting speed, but the distribution of the strain rate still follows the same rule; so, accurate quantitative research is still not possible, but rough quantitative research can be done for specific cutting speeds. Given that the mesh is processed by femtosecond laser, the size range is small; as such, only 10 meshes were used in this experiment. As shown in Figure 8a, the trends of the curve are in good agreement; that is, they gradually decrease from the beginning of the tool tip to the stagnation point of velocity and then increase. The tip strain rate is the highest because the tool tip deformation is the most severe. The velocity of the material at the stagnation point is the lowest, and the strain rate is the minimum. Figure $8 \mathrm{~b}$ shows the comparison of the strain rate distribution on the shear plane. The experimental and simulation results are all higher at the tip of the tool than at the middle part. In the experiment, the mesh is twisted and turned over because the material of the tool tip is seriously deformed (Figures 5 and 6). The image is blurred, which inevitably brings errors to the measurement; therefore, the initial data in the experiment are abnormal. In brief, regardless of the strain-rate distribution on the rake face or the distribution on the shear plane, the experimental distribution law is basically consistent with the calculated one. However, the experimental results and simulation should be further improved. First, the image processing technology needs to be improved because floating factors for the measurement of the grid distance are manually controlled by the operator. Second, further theoretical analysis and experimental confirmations should be conducted to study high-speed cutting from the point of view of flow.

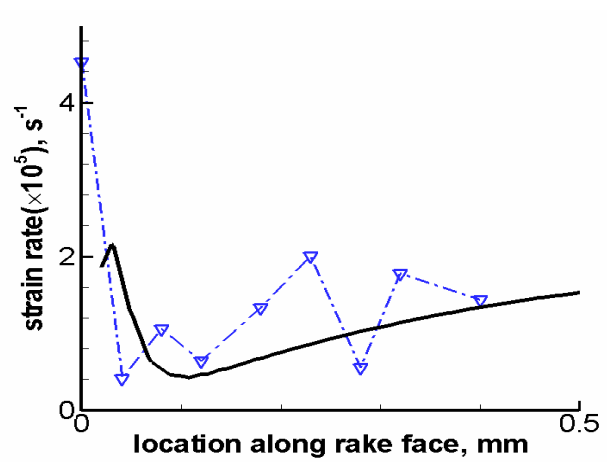

(a)

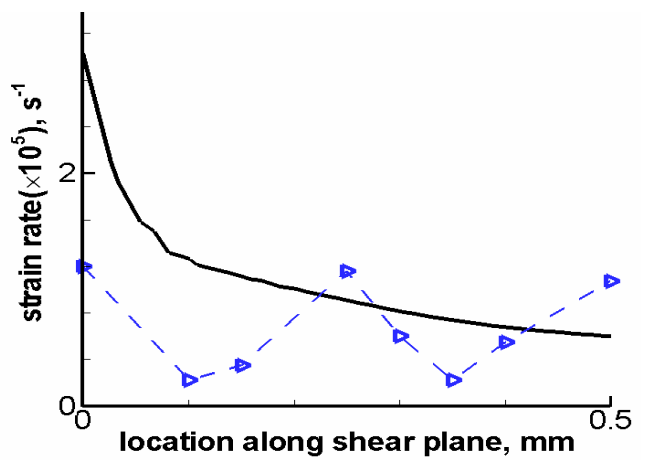

(b)

Figure 8. Strain rates obtained from calculations and experiments (dotted lines are the experiment results, and solid lines are the calculated results): (a) Comparison of the strain rate distribution on the rake face; (b) comparison of the strain rate distribution on the shear plane.

\section{Conclusions}

By analyzing the dislocation movement of the plastic deformation of metal materials, the theoretical basis of studying the metal cutting process from the angle of fluid flow is obtained. Through the fluid analysis of the cutting process and the verification of the cutting experiments, the following conclusions are obtained:

(1) The controlling mechanism of plastic deformation during the metal cutting process is the dislocation damping mechanism. The flow stress and strain rate in the deformation process are linearly related, which is also the theoretical basis for analyzing the metal cutting process from the perspective of fluid flow.

(2) In the cutting process, the strain rate can reach $10^{5}$ orders of magnitude, and the strain rate in the center of the first shear zone is larger than that in other regions along the shear plane direction. The two ends of the shear plane, i.e., the tip and the free surface, are the largest and then decrease from the inside to the outside. The closer to the rake face, the more severe the deformation and the greater the strain rate will be.

(3) From the point of view of flow, the properties of the cutting process can be obtained and quantitatively estimated. Although a large number of theoretical supporting and experimental results 
are needed, it does provide a new direction for the transition of strain-rate studies from qualitative research to quantitative research.

Author Contributions: K.Z. and Z.L. conceived and designed the experiments; K.W. performed the experiments; K.W. and X.X. analyzed the data; K.Z. wrote the paper. All authors have read and agreed to the published version of the manuscript.

Funding: This research was funded by the National Natural Science Foundation of China, grant number: [51775314, 51425503, 91860207].

Conflicts of Interest: The authors declare no conflict of interest.

\section{References}

1. Campbell, J.D.; Ferguson, W.G. The temperature and strain-rate dependence of the shear strength of mild steel. Philos. Mag. 1970, 81, 63-82. [CrossRef]

2. Jia, B.; Rusinek, A.; Pesci, R.; Bahi, S.; Bernier, R. Thermo-viscoplastic behavior of 304 austenitic stainless steel at various strain rates and temperatures: Testing, modeling and validation. Int. J. Mech. Sci. 2020, 170. [CrossRef]

3. He, N.; Lee, T.C. Assessment of deformation of a locallized chip in high speed machining. J. Mater. Process. Technol. 2002, 129, 101-104. [CrossRef]

4. List, G.; Sutter, G.; Bi, X.F.; Bouthiche, A. Strain, strain rate and velocity fields determination at very high cutting speed. J. Mater. Process. Technol. 2013, 213, 693-699. [CrossRef]

5. Rusinek, A.; Rodríguez-Martínez, J.A. Thermo-viscoplastic constitutive relation for aluminium alloys, modeling of negative strain rate sensitivity and viscous drag effects. Mater. Des. 2009, 30, 4377-4390. [CrossRef]

6. Kapoor, R.; Nemat-Nasser, S. Comparison between high and low strain-rate deformation of tantalum. Metall. Mater. Trans. A 2000, 31, 815-823. [CrossRef]

7. Hu, D.; Chen, M.H.; Wang, L.; Cheng, H. Dynamic tensile behaviour and deformational mechanism of C5191 phosphor bronze under high strain rates deformation. Mater. Sci. Eng. A 2016, 649, 68-73. [CrossRef]

8. Shaw, M.C. The size effect in metal cutting. Sädhanā 2003, 28, 875-896. [CrossRef]

9. El-zahry, R.M. On the hydrodynamic characteristics of the secondary shear zone in metal machining with sticking-sliding friction using the boundary layer theory. Wear 1987, 115, 349-359. [CrossRef]

10. Kwon, K.B.; Cho, D.W.; Lee, S.J. A fluid dynamic analysis model of the ultra-precision cutting mechanism. Ann. CLRP 1999, 48, 43-46. [CrossRef]

11. Müller, C.; Landua, S.; Blümke, R. Microstructure-a dominating parameter for chip forming during high speed milling. Mater. Und Werkst. 2015, 33, 194-199.

12. Thossatheppitak, B.; Uthaisangsuk, V.; Mungsuntisuk, P. Flow behaviour of Nickel Aluminum Bronze under hot deformation. Mater. Sci. Eng. A 2014, 604, 183-190. [CrossRef]

13. Lee, W.S.; Sue, W.C.; Lin, C.F. The strain rate and temperature dependence of the dynamic impact properties of 7075 aluminum alloy. J. Mater. Process. Technol. 2000, 100, 116-122. [CrossRef]

14. Kazban, R.V. Fluid mechanics approach to machining at high speeds: Part I: Justification of potential flow models. Mach. Sci. Technol. Int. J. 2007, 11, 475-489. [CrossRef]

15. Kazban, R.V. Fluid mechanics approach to machining at high speeds: Part II: A potential flow model. Mach. Sci. Technol. Int. J. 2007, 11, 491-514. [CrossRef]

16. Sundaram, N.K.; Guo, Y.; Chandrasekar, S. Mesoscale folding, instability, and disruption of laminar flow in metal surface. Phys. Rev. Lett. 2012, 109, 106001. [CrossRef]

17. Vandana, A.S.; Sundaram, N.K. Simulation of sinuous flow in metal cutting. Tribol. Lett. 2018, 66, 94-97. [CrossRef]

18. Meyers, M.A. Dynamic Behavior of Materials; John Wiley \& Sons, Inc.: New York, NY, USA, 1994; Volume 335.

19. Orowan, E. Problems of plastic gliding. Proc. Phys. Soc. 1940, 52, 8. [CrossRef]

20. Klocke, F.; Brockmann, M.; Gierlings, S.; Veselovac, D.; Kever, D.; Roidl, B.; Schmidt, G.; Semmler, U. Analytical modelling methods for temperature fields in metal cutting based on panel method of fluid mechanics. Procedia CIRP 2015, 31, 352-356. [CrossRef] 
21. Zhang, K.G.; Liu, Z.Q.; Wan, Y.; Wang, B.; Ai, X. Fluid-like properties of chip flow in high speed metal cutting process. Mach. Sci. Technol. 2015, 19, 71-85. [CrossRef]

22. Bahi, S.; List, G.; Sutter, G. Analysis of adhered contacts and boundary conditions of the secondary shear zone. Wear 2015, 330-331, 608-617. [CrossRef]

(c)

(C) 2020 by the authors. Licensee MDPI, Basel, Switzerland. This article is an open access article distributed under the terms and conditions of the Creative Commons Attribution (CC BY) license (http://creativecommons.org/licenses/by/4.0/). 